\title{
APPLICATION DES LOIS NON PARAMÉTRIQUES DANS LES SYSTÈMES D'ATTENTE ET LA THÉORIE DE RENOUVELLEMENT
}

\author{
Smail Adjabi ${ }^{1}$, KARIMA LAghA ${ }^{1}$ ET Amar AÏSsAni ${ }^{1}$ \\ Communiqué par Jean-Yves Jaffray
}

\begin{abstract}
Nonparametric survival distributions find, more and more, applications in a variety of field: reliability theory and survival analysis, queueing theory, maintenance, stock management, theory of the economy, ... The purpose of this paper is to use lower and upper bounds, in term of average means of the reliability functions belonging to the following distributions classes: IFR,DFR,NBU and $N W U$, which are presented by Sengupta (1994), to bound some of characteristics. We use some of these laws to evaluate the mean stationnary waiting time in $G I / G I / 1$ queueing system, by bringing up to date those bounds found by Stoyan (1983). As application to renewal theory and reliability, we use qualitative properties of time of repair to bound the mean of life system.
\end{abstract}

Résumé. Les distributions non paramétriques de survie trouvent, de plus en plus, des applications dans des domaines très variés, à savoir : théorie de fiabilité et analyse de survie, files d'attente, maintenance, gestion de stock, théorie de l'économie, ... L'objet de ce travail est d'utiliser les bornes inférieures et supérieures (en terme de la moyenne) des fonctions de fiabilité appartenant aux classes de distribution de type $I F R, D F R, N B U$ et $N W U$, présentées par Sengupta (1994), pour l'évaluation de certaines caractéristiques. Nous utilisons certaines de ces lois pour l'évaluation des bornes du temps moyen d'attente dans la file d'un système d'attente de type $G I / G I / 1$, en actualisant celles trouvées par Stoyan (1983). Comme application à la théorie de renouvellement et de fiabilité, nous utilisons les propriétés qualitatives des temps de réparation pour borner le temps moyen de vie d'un système à deux éléments réparables.

Mots Clés. Fiabilité, bornes, lois non paramétriques, temps de réparation, temps d'attente.

Received Janvier 7, 2002.

${ }^{1}$ Laboratoire de Modélisation et d'Optimisation des Systèmes LAMOS, Université de Béjaia, 06000 Béjaia, Algérie; e-mail : adjabi@hotmail.com

(C) EDP Sciences 2004 


\section{INTRODUCTION}

Le problème d'évaluation des performances d'un système d'attente se limite parfois à la recherche des bornes pour les caractéristiques en raison de la difficulté d'utilisation des résultats exacts existants $[4,5,12]$. Plusieurs méthodes d'approximation ont étés proposées (voir $[1,2,8-10,12]$ ). Certaines de ces approximations sont faites en utilisant les classes de distributions non paramétriques. Ces dernières trouvent des applications dans des domaines très variés tels que : la théorie de fiabilité et analyse de survie, files d'attente, maintenance, gestion de stock, théorie de l'économie, ...

Dans ce travail nous proposons l'utilisation de la propriété qualitative d'une distribution pour l'évaluation de certaines caractéristiques. Cette propriété permet de désigner la classe à laquelle appartient la distribution en question, à savoir : $I F R, N B U, D F R, N W U, \ldots$

À défaut d'informations suffisantes, cette propriété apporte souvent une première approximation dans les modèles de fiabilité $[3,6,7,11]$.

Dans la section 2, nous considérons la propriété de l'appartenance des distributions des temps des inter-arrivées, dans un système d'attente de type $G I / G I / 1$, à une classe de distribution non paramétrique donnée (IFR ou $N B U$ ).

La borne inférieure ou supérieure associée à la fonction fiabilité (voir Tab. $1^{1}$ ) est utilisée dans la relation (2). Cette relation exprime la propriété de monotonie externe [12] permettant de comparer deux systèmes d'attente, ce qui nous permet d'établir une borne inférieure ou supérieure pour le temps moyen d'attente dans la file du système d'attente $G I / G I / 1$. L'expression analytique exacte du temps moyen d'attente dans cette file est donnée dans [12] par :

$$
\mathbb{E} W=\frac{\left(m_{1}-m_{B}\right)^{2}+\sigma_{1}^{2}+\sigma_{B}^{2}}{2\left(m_{1}-m_{B}\right)}-\frac{m_{L}^{2}+\sigma_{L}^{2}}{2 m_{L}}
$$

où $m_{L}, m_{1}$ et $m_{B}$ sont respectivement les moyennes de la période d'inoccupation, du temps des inter-arrivées et du temps de service. $\sigma_{L}^{2}, \sigma_{1}^{2}$ et $\sigma_{B}^{2}$ sont les variances associées.

Dans la section 3, on considère un système formé de deux éléments réparables dont la distribution des temps de réparation appartient à une classe de distributions non paramétrique donnée $(I F R, N B U, D F R$ et $N W U)$ et les temps de fonctionnement sont exponentiellement distribués.

Sous la condition de comparaison exprimée dans la proposition 7.2.1 de la référence [12] (voir la relation (5)), les résultats présentés dans le tableau 1 vont nous permettre d'établir une borne inférieure ou supérieure pour le temps moyen de vie du système.

\footnotetext{
${ }^{1}$ La méthode permettant d'évaluer ces bornes est présentée en détail par Sengupta dans [11].
} 
TABleau 1. Bornes de $\bar{F}(x)$ (basées sur le $r^{e}$ moment $m_{r}$ ) dans différents cas.

\begin{tabular}{|c|c|c|}
\hline Classe & Borne supérieure & Borne inférieure \\
\hline$I F R$ & $\begin{array}{c}\bar{F}(x) \leq\left\{\begin{array}{ccc}1 & \text { si } & x<m_{r}^{1 / r} \\
\delta_{x} & \text { si } & x \geq m_{r}^{1 / r}\end{array}\right. \\
\text { où }\left[\int_{0}^{1} r y^{r-1} \delta_{x}^{y} \mathrm{~d} y=\frac{m_{r}}{x^{r}}\right]\end{array}$ & $\begin{array}{r}\bar{F}(x) \geq\left\{\begin{array}{ccc}\inf _{0 \leq \beta \leq x} \mathrm{e}^{-\alpha} & \text { si } & x<m_{r}^{1 / r} \\
0 & \text { si } & x \geq m_{r}^{1 / r}\end{array}\right. \\
\text { où }\left[\int_{0}^{\infty}\left(\beta+\frac{x-\beta}{\alpha} z\right)^{r} \mathrm{e}^{-\alpha} \mathrm{d} z=m_{r}\right]\end{array}$ \\
\hline$N B U$ & $\begin{array}{c}\bar{F}(x) \leq\left\{\begin{array}{ccc}1 & \text { si } & x<m_{r}^{1 / r} \\
\delta_{x} & \text { si } & x \geq m_{r}^{1 / r}\end{array}\right. \\
\text { où }\left[\int_{0}^{1} r y^{r-1} \delta_{x}^{y} \mathrm{~d} y=\frac{m_{r}}{x^{r}}\right]\end{array}$ & $\begin{array}{c}\bar{F}(x) \geq\left\{\begin{array}{ccc}\delta_{x} & \text { si } \quad x<m_{r}^{1 / r} \\
0 & \text { si } \quad x \geq m_{r}^{1 / r}\end{array}\right. \\
\text { où }\left[\sum_{j=0}^{\infty} \delta_{x}^{j}\left[(j+1)^{r}-j^{r}\right]=\frac{m_{r}}{x^{r}}\right]\end{array}$ \\
\hline$D F R$ & $\begin{array}{c}\bar{F}(x) \leq\left\{\begin{array}{cc}\mathrm{e}^{-\left(r x / x_{0}\right)} & \text { si } x<x_{0} \\
\left(x_{0} / x\right)^{r} \mathrm{e}^{-r} & \text { si } x \geq x_{0}\end{array}\right. \\
\text { où } x_{0}=r\left[\frac{m_{r}}{\Gamma(r+1)}\right]^{1 / r}\end{array}$ & $\bar{F}(x) \geq 0$ \\
\hline$N W U$ & $\begin{array}{c}\bar{F}(x) \leq \delta_{x} \\
\text { où } \sum_{j=1}^{\infty} \delta_{x}^{j}\left[j^{r}-(j-1)^{r}\right]=\frac{m_{r}}{x^{r}}\end{array}$ & $\bar{F}(x) \geq 0$ \\
\hline
\end{tabular}

L'expression analytique de ce dernier en fonction des temps de réparation est donnée dans [12] par la relation suivante :

$$
\mathbb{E} T=\lambda^{-1}\left(1+\left(1-\int_{0}^{\infty} \mathrm{e}^{-\lambda t} \mathrm{~d} R(t)\right)^{-1}\right),
$$

où $\lambda$ et $R$ désignent respectivement le paramètre des temps de fonctionnement et la distribution des temps de réparation.

\section{Application au système D'Attente $G I / G I / 1$}

Soit $F$ une distribution de vie définie sur $[0, \infty)$. On note par $\bar{F}(t)=1-F(t)$ la fonction fiabilité associée. Les bornes proposées par Sengupta [11] dans le cas de l'appartenance de la distribution $F$ à une classe non paramétrique, telles que : IFR, $N B U, D F R$ ou $N W U$, sont présentées dans le tableau 1.

Étant donnés deux systèmes $A_{1} / B_{1} / 1$ (dit original) et $A_{2} / B_{2} / 1$ (dit système d'approximation), où $A_{i}$ représente la distribution des temps des inter-arrivées de moyenne $m_{i}$ finie, de variance $\sigma_{i}^{2}$ et de coefficient de variation $C_{a i}=\sigma_{i} / m_{i}$ et $B_{i}$ la distribution de service dans le système $A_{i} / B_{i} / 1$ d'intensité de trafic $\rho_{i}, i=1,2$.

On suppose que les deux systèmes possèdent une discipline de service FIFO (First In First Out), une capacité infinie et que les processus d'arrivée et de service 
sont des processus de renouvellements. Notons $m_{W i}, i=1,2$, le temps moyen d'attente dans la file.

Le théorème 5.2.1 [12] énonce la propriété de monotonie externe. Cette propriété permet sous la condition suffisante suivante :

$$
A_{2} \leq_{c v} A_{1} \text { et } B_{1} \leq_{c} B_{2},
$$

de conclure que $m_{W 1} \leq m_{W 2}$, où $\leq_{c}\left(\right.$ resp. $\left.\leq_{c v}\right)$ désigne l'ordre convexe (resp. concave).

On suppose que le système original possède la même distribution de service que le système d'approximation tel que le second moment $\mathbb{E} \beta^{2}$ est fini.

Soit $B$ la distribution de service, la moyenne et la variance associées à cette distribution sont notées par $m_{B}$ et $\sigma_{B}^{2}$, respectivement, d'où $\rho_{i}=m_{B} / m_{i}, i=1,2$.

Si la distribution $A_{1}$ possède une propriété qualitative donnée (IFR ou $N B U$ ), alors elle est majorée ou minorée par la distribution $A_{2}$. Cette dernière est exprimée dans le tableau 1. En utilisant cette propriété nous proposons une borne supérieure (dans chaque cas) pour le temps moyen d'attente stationnaire dans la file du système $G I / G I / 1(\infty, F I F O)$.

\subsection{Distribution IFR}

On suppose que la distribution $A_{1}$ appartient à la classe $I F R$ « Increasing Failure Rate ». À partir de la borne inférieure correspondant à la distribution IFR (voir Tab. 1) et en utilisant la relation (2), on établit une borne supérieure pour le temps moyen d'attente dans la file, donnée par :

$$
\frac{\sigma_{1}^{2}+\sigma_{B}^{2}}{2 m_{1}\left(1-\rho_{1}\right)}-1 / 2 m_{1}\left(\rho_{1}+C_{a 1}^{2}\right) \leq m_{W 1} \leq \frac{\mathbb{E} \beta^{2}}{2 m_{1}\left[1-\mathrm{e}^{-1}-\rho_{1}\right]}
$$

Démonstration. Si $A_{1}$ est une distribution IFR, alors $\forall r>0$,

$$
\overline{A_{1}}(x) \geq \bar{A}_{2}(x)=\left\{\begin{array}{cc}
\mathrm{e}^{-\alpha x} & \text { si } x<m_{1 r}^{1 / r} \\
0 & \text { si } x \geq m_{1 r}^{1 / r}
\end{array} \quad \text { où } \quad \alpha=\left[\frac{\Gamma(r+1)}{m_{1 r}}\right]^{1 / r},\right.
$$

avec $m_{1 r}$ désigne le $r^{e}$ moment de la distribution $A_{1}$. Par suite, $A_{2} \leq s t A_{1}$ donc $A_{2} \leq_{c v} A_{1}$, où $\leq_{s t}$ désigne l'ordre stochastique. La condition suffisante exprimée dans la relation (2) est alors vérifiée d'où $m_{W 1} \leq m_{W 2}$. Il suffit donc de calculer le temps moyen d'attente $m_{W 2}$ dans la file du système $\left(\begin{array}{ccc}G I / & G I / & 1 \\ A_{2} & B & \end{array}\right)$.

Le taux de défaillance associé à la distribution $A_{2}$ est défini par $\lambda(t)=$ $\lim _{x \rightarrow 0}\left(\frac{\overline{A_{2}}(t)-\overline{A_{2}}(t+x)}{x \overline{A_{2}}(t)}\right)$. Par conséquent, $\lambda(t)=\alpha$ sur l'intervalle $\left[0, m_{1 r}^{1 / r}[\right.$ et est non défini pour $t \geq m_{1 r}^{1 / r}$. La distribution $A_{2}$ est donc exponentielle sur l'intervalle $\left[0, m_{1 r}^{1 / r}\right.$ [ et de moyenne $m_{2}=\int_{0}^{+\infty} \bar{A}_{2}(x) \mathrm{d} x=\frac{1}{\alpha}\left(1-\mathrm{e}^{-\alpha m_{1 r}^{1 / r}}\right)<\infty$. 
Le temps moyen d'attente $m_{W 2}$ dans la file du système $A_{2} / B / 1$ est donc égal à :

$$
m_{W 2}=\frac{\sigma_{B}^{2}+m_{B}^{2}}{2 m_{2}\left(1-\rho_{2}\right)}=\frac{\mathbb{E} \beta^{2}}{\frac{2}{\alpha}\left(1-\mathrm{e}^{-\alpha m_{1 r}^{1 / r}}\right)\left(1-\rho_{2}\right)} .
$$

Pour $r=1$, on a $\alpha=\frac{1}{m_{1}}$ et $m_{2}=m_{1}\left(1-\mathrm{e}^{-1}\right)$ avec $\rho_{1}=\frac{m_{B}}{m_{1}}$, d'où

$$
m_{W 1} \leq m_{W 2}=\frac{\mathbb{E} \beta^{2}}{2 m_{1}\left(1-\mathrm{e}^{-1}-\rho_{1}\right)} .
$$

\subsection{Distribution $N B U$}

En utilisant la borne inférieure de la fonction fiabilité correspondant à la distribution $A_{1}$ de loi $N B U$ « New Better than Used $»$ (voir Tab. 1) et la relation (2), nous établissons une borne supérieure pour le temps moyen d'attente dans la file, donnée par :

$$
\frac{\sigma_{1}^{2}+\sigma_{B}^{2}}{2 m_{1}\left(1-\rho_{1}\right)}-1 / 2 m_{1}\left(\rho_{1}+1\right) \leq m_{W 1} \leq \frac{\mathbb{E} \beta^{2}}{m_{1}\left[1-\mathrm{e}^{-1}-2 \rho_{1}\right]} .
$$

Démonstration. Si $A_{1}$ est une distribution $N B U$, alors $\forall r>0$

$$
\bar{A}_{1}(x) \geq \bar{A}_{2}(x)=\left\{\begin{array}{cl}
\delta_{x} & x<m_{1 r}^{1 / r} \\
0 & x \geq m_{1 r}^{1 / r}
\end{array}\right.
$$

où $\delta_{x}$ est une fonction de $x$ et de $m_{1 r}$ telle que $\sum_{j=0}^{\infty} \delta_{x}^{j}\left[(j+1)^{r}-j^{r}\right]=\frac{m_{1 r}}{x^{r}}$.

$$
\begin{gathered}
\text { Si } r=1, \quad \text { alors } \delta_{x}=\left\{\begin{array}{cc}
1-\frac{x}{m_{1}} & \text { si } x<m_{1} \\
0 & \text { sinon }
\end{array}\right. \text {, d'où } \\
\bar{A}_{2}(x)=\left\{\begin{array}{cc}
1-\frac{x}{m_{1}} & \text { si } x<m_{1} \\
0 & x \geq m_{1} .
\end{array}\right.
\end{gathered}
$$

On a alors $A_{2}<_{s t} A_{1}$ donc $A_{2}<_{c v} A_{1}$, d'où la condition exprimée par la relation (2). Par conséquent, $m_{W 1} \leq m_{W 2}$. Le taux de défaillance pour la fonction $A_{2}$ est alors $\lambda(t)=\frac{1}{m_{1}-t} \cdot \mathbf{1}_{\left[0, m_{1}[\right.}[t)$, où $\mathbf{1}_{\left[0, m_{1}[\right.}$ désigne la fonction Indicatrice sur $\left[0, m_{1}\left[\right.\right.$, d'où $\lambda(t)$ est une fonction croissante sur [0, $m_{1}\left[\right.$. Donc $A_{2}$ est une distribution $I F R$, de moyenne $m_{2}=m_{1} / 2<\infty$. Le temps moyen d'attente $m_{W 2}$ correspondant au système $A_{2} / B / 1$ avec $A_{2}$ une distribution $I F R$ est alors majoré par la borne supérieure de la relation (3). On a donc 


$$
\begin{aligned}
& m_{W 1} \leq m_{W 2} \leq \frac{\left(\sigma_{B}^{2}+m_{B}^{2}\right)}{2 m_{2}\left[1-\mathrm{e}^{-1}-\rho_{2}\right]}, \quad \text { avec } \quad \begin{cases}m_{2}= & \frac{m_{1}}{2} \\
\rho_{2}= & 2 \rho_{1}\end{cases} \\
& \text { D'où, } \quad m_{W 1} \leq \frac{\mathbb{E} \beta^{2}}{m_{1}\left(1-\mathrm{e}^{-1}-2 \rho_{1}\right)} \text {. }
\end{aligned}
$$

TABleau 2. Borne supérieure de la quantité $\frac{m_{1}}{\mathbb{E} \beta^{2}} \cdot m_{W 1}$.

\begin{tabular}{|l|c|c|c|c|c|c|}
\hline Classe $\backslash \rho_{1}$ & 0,1 & 0,2 & 0,3 & 0,4 & 0,5 & 0,6 \\
\hline$I F R$ & 0,939 & 1,157 & 1,505 & 2,154 & 3,784 & 15,566 \\
\hline$N B U$ & 2,3142 & 4,3081 & 31,1327 & $\times$ & $\times$ & $\times$ \\
\hline
\end{tabular}

\subsection{RÉsultats}

- La borne supérieure proposée dans le cas d'une distribution IFR (voir la relation $(3))$ existe sous la condition $\rho_{1}<1-\mathrm{e}^{-1} \simeq 0,6321$ et les valeurs de la borne associée à la quantité $\frac{m_{1}}{\mathbb{E} \beta^{2}} \cdot m_{W 1}$ sont données dans la première ligne du tableau 2.

- La borne supérieure proposée dans le cas d'une distribution $N B U$ (voir la relation (4)) existe si $\rho_{1}<\left(\frac{1-\mathrm{e}^{-1}}{2}\right) \simeq 0,316$ et les valeurs de la borne associée à la quantité $\frac{m_{1}}{\mathbb{E} \beta^{2}} \cdot m_{W 1}$ sont données dans la deuxième ligne du tableau 2.

- Les résultats sont présentés dans le tableau 2 pour différentes valeurs de $\rho_{1}$.

- La borne supérieure proposée dans chacun des deux cas (IFR et $N B U)$ est une fonction croissante du taux de trafic. Ceci apparaît clairement dans les résultats du tableau 2.

- D'après les résultats de ce tableau, le temps moyen d'attente $m_{W 1}$ dans le cas $I F R$ reste relativement faible pour les valeurs de $\rho_{1}$ variant de 0,1 à 0,5 mais augmente rapidement dès que $\rho_{1}$ dépasse 0,5 .

Dans le cas $N B U$, le temps moyen d'attente dans la file du système devient important dès que $\rho_{1}$ dépasse 0,2 . On note que le maximum obtenu pour cette valeur dépasse largement celui atteint dans le cas IFR.

Remarque 1. La borne inférieure de la relation (3) (donnée dans le cas IFR) est la borne proposée par Kleinrock [8]. Cette même borne est proposée par Stoyan [12] dans le cas d'une distribution $D M R L$ « Decreasing Mean Residual Life ». Elle reste donc valable dans le cas $I F R$ en raison de la relation $I F R \Rightarrow D M R L$.

Elle est proposée par Stoyan [12] dans le cas d'une distribution $N B U E \ll$ New Better than Used in Expectation » avec $C_{a}^{2}$ remplacée par 1. Elle reste donc valable car $I F R \Rightarrow N B U \Rightarrow N B U E$. D'où la borne inférieure de l'inégalité (4) correspondante au cas $N B U$. 
Remarque 2. Dans le reste des cas, il paraît difficile d'obtenir des bornes, en raison de :

- La difficulté d'obtention de la fonction $\delta_{x}$ dans les bornes supérieures pour les classes $I F R, N B U$ et $N W U$ « New Worse than Used », ce qui ne permet pas la détermination de la distribution $A_{2}$.

- L'appartenance de la fonction borne à la même classe de distributions pour le cas $D F R$ « Decreasing Failure Rate».

La borne inférieure de la relation (4) correspondante au cas $N B U E$ (voir Rem. 1) est une borne supérieure dans le cas d'une distribution $N W U E$ [12]. Elle est donc une borne supérieure pour une distribution $D F R$ ou $N W U$ (car $D F R \Rightarrow N W U \Rightarrow$ NWUE).

\section{ApplicAtion À la thÉORIE DE RENOUVELlement}

Considérons un système formé de deux éléments réparables $\xi_{1}$ et $\xi_{2}$. À l'instant $t=0$, l'élément $\xi_{1}$ fonctionne jusqu'à la défaillance à la date $t=X_{0}$ où la réparation commence à s'effectuer sur cet élément et prend un temps égal à $Y_{1}$, alors que $\xi_{2}$ commence à fonctionner jusqu'à sa défaillance à la date $X_{1}$. Si $X_{1} \leq Y_{1}$, le système s'arrête à la date $X_{0}+X_{1}$. Sinon, $\xi_{1}$ fonctionne encore à la date $X_{0}+X_{1}$ alors que la réparation de $\xi_{2}$ est commencée.

Les temps de fonctionnement $X_{0}, X_{1}, \ldots$ sont supposés indépendants identiquement distribués et indépendants des temps de réparations successives $Y_{1}, Y_{2}, \ldots$, qui sont aussi indépendants, identiquement distribués, de moyenne d'ordre $r$ notée $m_{r}, r \geq 1$, de variance $\sigma_{R}^{2}$ et de coefficient de variation $C_{R}=\sigma_{R} / m_{1}$.

On définit $N=\inf \left\{n: X_{n}<Y_{n}\right\}$. Le temps de vie $T$ du système est la variable aléatoire $T=X_{0}+X_{1}+\ldots+X_{N}$.

On suppose que les temps de fonctionnement $X_{i}$ sont exponentiellement distribués, de paramètre $\lambda$ et que les temps de réparation ont une fonction distribution $R$ appartenant à une classe de distributions non paramétrique donnée.

Le temps moyen de vie du système est exprimé en fonction des temps de réparation par la relation (1).

La proposition 7.2.1 énoncée dans [12] permet la comparaison du temps moyen de vie de deux systèmes identiques à deux éléments réparables (tels que décrits ci-dessus) ayant pour fonctions de distribution des temps de réparation les fonctions $R_{1}$ et $R_{2}$ et tels que $\lambda_{1}$ et $\lambda_{2}$ sont les paramètres des temps de fonctionnement. Sous la condition suivante :

$$
\lambda_{1} \leq \lambda_{2} \quad \text { et } \quad R_{1}<{ }_{L} R_{2},
$$

où $<_{L}$ désigne l'ordre Laplacien.

Les temps de vie moyens des deux systèmes sont tels que $\mathbb{E} T_{1} \geq \mathbb{E} T_{2}$. Stoyan [12] propose les deux résultats suivants :

$$
1+\left(1-\mathrm{e}^{-\lambda m_{1}}\right)^{-1} \leq \lambda \mathbb{E} T \leq 1+\left(1+C_{R}^{2}\right) /\left(1-\exp \left(-\lambda m_{1}\left(1+C_{R}^{2}\right)\right)\right),
$$


et si $R$ est $N B U E$, alors

$$
1+\left(1-\mathrm{e}^{-\lambda m_{1}}\right)^{-1} \leq \lambda \mathbb{E} T \leq 1+\left(1+\lambda m_{1}\right)^{-1} .
$$

On suppose dans toute la suite que $\lambda_{1}=\lambda_{2}=\lambda$. La distribution des temps de réparation $R_{1}$ est majorée ou minorée par la distribution $R_{2}$. Cette dernière est exprimée dans le tableau 1 suivant la propriété qualitative de $R_{1}$ (IFR, NBU, $D F R$ ou $N W U)$. En utilisant cette propriété, nous évaluons les bornes inférieures ou supérieures pour le temps moyen de vie du système.

\subsection{TEMPS DE RÉPARATION IFR}

On suppose que la distribution $R_{1}$ appartient à la classe $I F R$ « Increasing Failure Rate ». Â partir de la borne inférieure correspondante à la distribution IFR (voir Tab. 1) et en utilisant la relation (5), on établit une borne inférieure pour le temps moyen de vie du système exprimé par la relation (1). Cette borne est donnée par :

$$
1+(1+\beta)^{-1} \geq \lambda \mathbb{E} T_{1} \geq\left[1+\frac{\beta^{-1}+1}{1-\mathrm{e}^{-(1+\beta)}}\right], \quad \text { avec } \beta=\lambda m_{1}
$$

Démonstration. Si $R_{1}$ est IFR, alors $\forall r>0$,

$$
\bar{R}_{1}(x) \geq \bar{R}_{2}(x)=\left\{\begin{array}{cl}
\mathrm{e}^{-\alpha x} & \text { si } x<m_{r}^{1 / r} \\
0 & \text { si } x \geq m_{r}^{1 / r}
\end{array} \quad \alpha=\left[\frac{\Gamma(r+1)}{m_{r}}\right]^{1 / r} .\right.
$$

On a alors $R_{1}>_{s t} R_{2}$. Donc $R_{2}<_{L} R_{1}$, d'où la condition (5). Par conséquent, pour toute fonction $f$ monotone et dérivable, $\int_{0}^{\infty} f(x) \mathrm{d} R_{2}(x) \leq \int_{0}^{\infty} f(x) \mathrm{d} R_{1}(x)$. En particulier, si $f(x)=\mathrm{e}^{-\lambda x}$, on a :

$$
\int_{0}^{\infty} \mathrm{e}^{-\lambda x} \mathrm{~d} R_{2}(x) \leq \int_{0}^{\infty} \mathrm{e}^{-\lambda x} \mathrm{~d} R_{1}(x)
$$

d'où,

$$
\int_{0}^{\infty} \mathrm{e}^{-\lambda x} \mathrm{~d} R_{1}(x) \geq \frac{1}{\beta+1}\left[1+\beta \mathrm{e}^{-\alpha(1+\beta) m_{r}^{1 / r}}\right], \quad \text { avec } \beta=\frac{\lambda}{\alpha}
$$

Par conséquent,

$$
\mathbb{E} T_{1} \geq \lambda^{-1}\left[1+\frac{1+\beta^{-1}}{1-\mathrm{e}^{-(1+\beta)(\Gamma(r+1))^{1 / r}}}\right], \quad \beta=\frac{\lambda}{\alpha} \text { et } r>0 .
$$

Le résultat est obtenu pour $r=1$. 


\subsection{TEMPs DE RÉPARATION $N B U$}

En utilisant la borne inférieure de la fonction fiabilité correspondante à la distribution $R_{1}$ de loi $N B U$ « New Better than Used» (Tab. 1) et la relation (5), on établit une borne inférieure pour le temps moyen de vie du système (exprimé par la relation (1)), donnée par :

$$
1+(1+\beta)^{-1} \geq \lambda \mathbb{E} T_{1} \geq 1+\frac{\beta}{\beta+\mathrm{e}^{-\beta}-1}, \quad \beta=\lambda m_{1} .
$$

Démonstration. Si $R_{1}$ est $N B U$, alors pour $r=1$

$$
\overline{R_{1}}(x) \geq \overline{R_{2}}(x)=\left\{\begin{array}{ccc}
1-\frac{x}{m_{1}} & \text { si } x<m_{1} \\
0 & \text { si } x \geq m_{1}
\end{array}\right.
$$

D'où, $R_{2}<_{s t} R_{1}$. Donc, $R_{2}<_{L} R_{1}$. En utilisant la relation (7) on obtient,

$$
\int_{0}^{\infty} \mathrm{e}^{-\lambda x} \mathrm{~d} R_{1}(x) \geq \frac{1}{\beta}\left(1-\mathrm{e}^{-\beta}\right), \quad \text { où } \beta=\lambda m_{1} .
$$

D'où,

$$
\mathbb{E} T_{1} \geq \lambda^{-1}\left[1+\frac{\beta}{\beta+\mathrm{e}^{-\beta}-1}\right]
$$

\subsection{TEMPS DE RÉPARATION $D F R$}

En utilisant la borne supérieure de la fonction fiabilité correspondante à la distribution $R_{1}$ de loi $D F R$ « Decreasing Failure Rate (Tab. 1) et la relation (5), on établit une borne supérieure pour le temps moyen de vie du système, donnée par :

$$
\begin{array}{r}
1+\left(1-\mathrm{e}^{-\lambda m_{1}}\right)^{-1} \leq \lambda \mathbb{E} T_{1} \leq\left[1+\frac{r+\theta}{\theta\left(1-\mathrm{e}^{-(r+\theta)}\right)+(r+\theta) \mathrm{e}^{-r} \theta^{r} \int_{\theta}^{\infty} x^{-r} \mathrm{e}^{-x} \mathrm{~d} x}\right] \\
\theta=\lambda x_{0} .
\end{array}
$$

Démonstration. Si $R_{1}$ est $D F R$, alors,

$$
\bar{R}_{1}(x) \leq \bar{R}_{2}(x)=\left\{\begin{array}{cl}
\mathrm{e}^{-\left(r x / x_{0}\right)}, & x<x_{0} \\
\left(x_{0} / x\right)^{r} \mathrm{e}^{-r}, & x \geq x_{0},
\end{array} \quad \text { tel que } x_{0}=r\left[\frac{m_{r}}{\Gamma(r+1)}\right]^{1 / r} .\right.
$$

D'où $R_{1}<_{s t} R_{2}$. Donc, $R_{1}<_{L} R_{2}$. On a alors

$$
\int_{0}^{\infty} \mathrm{e}^{-\lambda x} \mathrm{~d} R_{1}(x) \leq \int_{0}^{\infty} \mathrm{e}^{-\lambda x} \mathrm{~d} R_{2}(x) .
$$


D'où, $\int_{0}^{\infty} \mathrm{e}^{-\lambda x} \mathrm{~d} R_{1}(x) \leq \frac{1}{r+\lambda x_{0}}\left(\lambda x_{0} \mathrm{e}^{-\left(r+\lambda x_{0}\right)}+r\right)-\lambda x_{0}^{r} \mathrm{e}^{-r} \int_{x_{0}}^{+\infty} x^{-r} \mathrm{e}^{-\lambda x} \mathrm{~d} x$.

Par conséquent, pour tout $r>0$,

$$
\mathbb{E} T_{1} \leq \lambda^{-1}\left[1+\frac{r+\theta}{\theta\left(1-\mathrm{e}^{-(r+\theta)}\right)+(r+\theta) \mathrm{e}^{-r} \theta^{r} \int_{\theta}^{\infty} x^{-r} \mathrm{e}^{-x} \mathrm{~d} x}\right], \quad \theta=\lambda x_{0}
$$

Le résultat est obtenu pour $r=1$.

\subsection{TEMPS DE RÉPARATION $N W U$}

En utilisant la borne supérieure de la fonction fiabilité correspondant à la distribution $R_{1}$ de loi $N W U$ « New Worse thand Used» (Tab. 1) et la relation (5), on établit une borne supérieure pour le temps moyen de vie du système, donnée par :

$$
1+\left(1-\mathrm{e}^{-\lambda m_{1}}\right)^{-1} \leq \lambda \mathbb{E} T_{1} \leq\left[1+\frac{1}{1-\theta \mathrm{e}^{\theta} \int_{\theta}^{\infty} \mathrm{e}^{-x} x^{-2} \mathrm{~d} x}\right], \quad \theta=\lambda m_{1}
$$

Démonstration. Si $R_{1}$ est $N W U$, alors pour $r=1$ on a $\bar{R}_{1}(x) \leq \bar{R}_{2}(x)=$ $\frac{m_{1}}{m_{1}+x}, x>0$. D'où, $R_{1}<_{s t} R_{2}$. Donc, $R_{1}<_{L} R_{2}$. En utilisant la relation (8) on obtient,

$$
\int_{0}^{\infty} \mathrm{e}^{-\lambda x} \mathrm{~d} R_{1}(x) \leq m_{1} \int_{0}^{+\infty} \frac{\mathrm{e}^{-\lambda x}}{\left(m_{1}+x\right)^{2}} \mathrm{~d} x
$$

Par conséquent,

$$
\mathbb{E} T_{1} \leq \lambda^{-1}\left[1+\frac{1}{1-\theta \mathrm{e}^{\theta} \int_{\theta}^{\infty} \mathrm{e}^{-x} x^{-2} \mathrm{~d} x}\right], \quad \theta=\lambda m_{1}
$$

Remarque 3. La borne supérieure de la relation (6) correspondant au cas $N B U E$ est aussi une borne supérieure dans le cas de la distribution des temps de réparation appartenant à la classe $I F R$ ou $N B U$. Ceci est justifiée par la relation suivante :

$$
I F R \Rightarrow N B U \Rightarrow N B U E
$$

Remarque 4. On vérifie aisément que les bornes inférieures que nous avons proposées dans cette partie, pour les temps moyens de vie d'un système à deux éléments réparables, dans le cas $I F R$ et $N B U$ sont plus fines que celles proposées par Stoyan [12].

Remarque 5. La borne inférieure de la relation (6) est une borne proposée par Stoyan pour n'importe quelle distribution des temps de réparation. On peut donc l'utiliser dans le cas $D F R$ et $N W U$. 
Remarque 6. Dans le cas des distributions DFR et $N W U$, les bornes supérieures que nous avons proposées sont fonction de l'intégrale :

$$
\int_{\theta}^{\infty} \mathrm{e}^{-y} y^{-r} \mathrm{~d} y, \text { avec } \theta=\lambda m_{r} .
$$

Cette intégrale est convergente pour toute valeur de $r$ positive.

\section{Conclusion}

Dans ce travail nous avons traité le problème d'évaluation de performances d'un système d'attente de type $G I / G I / 1$ et d'un système formé de deux éléments réparables, en utilisant des distributions non paramétriques. Nous avons considéré la propriété qualitative de la distribution des temps des inter-arrivées pour le premier système et celle de la distribution des temps de réparation pour le deuxième système.

Les caractéristiques étudiées sont le temps moyen d'attente dans la file du premier système et le temps moyen de vie dans le deuxième système. On note que dans ce cas, nous obtenons des bornes pour ces caractéristiques. La propriété qualitative est utilisée à défaut d'information suffisante et elle apporte souvent une première approximation dans les modèles de fiabilité $[3,6,7,11]$.

Les bornes proposées dans cet article peuvent être utilisées pour d'autres classes de distributions : dans le premier système étudié, la borne supérieure proposée pour le temps moyen d'attente dans le cas $N B U$ (relation (4)) peut être utilisée pour la classe IFRA « Increasing Failure Rate on the Average », car IFRA $\Rightarrow N B U$.

Dans le deuxième système considéré, on peut utiliser les bornes proposées pour d'autres classes de distributions des temps de réparation, en tenant compte des relations suivantes :

$$
I F R \Rightarrow I F R A \Rightarrow N B U \text { et } \quad D F R \Rightarrow D F R A \Rightarrow N W U
$$

\section{RÉFÉRENCES}

[1] I.J.B.F. Adan, W.A. Van de Waarsenburg and J. Wessels, Analyzing $E_{k} / E_{r} / c$ Queues. EJOR 92 (1996) 112-124.

[2] A. Aïssani, Comparing due-date-based performance measures for queueing models. Belg. J. Oper. Res. Statist. Comput. Sci. 39 (1999) 2-3.

[3] R.E. Barlow and F. Barlow, Mathematical Theory of Reliability. John Wiley, New York (1965).

[4] D. Bertsimas, An exact FCFS waiting time analysis for a class of G/G/s queueing systems. Queue. Syst. Theor. Appl. 3 (1988) 305-320.

[5] D. Bertsimas, An analytic approach to a general class of $\mathrm{G} / \mathrm{G} / \mathrm{s}$ queueing systems. Oper. Res. 38 (1990) 139-155.

[6] J.L. Bon, Fiabilité des systèmes : Méthodes mathématiques. Masson, Paris (1995).

[7] G. Chaudhuri, J.V. Deshpande and A.D. Dharmadhikari, Some bounds on reliability of coherent systems of IFRA composants. J. Appl. Probab. 28 (1991) 709-714. 
[8] L. Kleinrock, Queueing systems. Technical report, Computer applications. John Wiley and Sons (1976 II).

[9] Shun-Chen Niu, On the comparison of waiting times in tandem queues. J. Appl. Probab. 18 (1981) 707-714.

[10] G. Pujolle and S. Fdida, Modèles de systèmes et de réseaux (files d'attente), Vol. 1. Eyrolle 1 (1989).

[11] D. Sengupta, Another look at the moment bounds on reliability. J. Appl. Probab. 31 (1994) $777-787$.

[12] D. Stoyan, Comparison methods for queueing models and other stochastic models. John Wiley (1983).

To access this journal online: www.edpsciences.org 